\title{
Min-wise independent families with respect to any linear order
}

\author{
Peter J. Cameron and Pablo Spiga ${ }^{* \dagger}$ \\ School of Mathematical Sciences \\ Queen Mary, University of London \\ Mile End Road \\ London, E1 4NS \\ U.K.
}

\begin{abstract}
A set of permutations $\mathscr{S}$ on a finite linearly ordered set $\Omega$ is said to be $k$-min-wise independent, $k$-MWI for short, if $\operatorname{Pr}(\min \pi(X)=\pi(x))=1 /|X|$ for every $X \subseteq \Omega$ such that $|X| \leq k$ and for every $x \in X$. (Here $\pi(x)$ and $\pi(X)$ denote the image of the element $x$ or subset $X$ of $\Omega$ under the permutation $\pi$, and $\operatorname{Pr}$ refers to a probability distribution on $\mathscr{S}$, which we take to be the uniform distribution.) We are concerned with sets of permutations which are $k$-MWI families for any linear order. Indeed, we characterize such families in a way that does not involve the underlying order. As an application of this result, and using the Classification of Finite Simple Groups, we deduce a complete classification of the $k$-MWI families that are groups, for $k \geq 3$.
\end{abstract}

\section{Introduction}

We let $\operatorname{Sym} \Omega$ and Alt $\Omega$ denote the symmetric group and the alternating group on the set $\Omega$ respectively. If $k$ is a natural number then $\operatorname{Sym}(k)$ will denote the

\footnotetext{
* Supported by a grant from the Istituto Nazionale di Alta Matemati ca, INdAM

† Current address: Department of Mathematics, University of Lethbridge, 4401 University Drive, Lethbridge, Alberta, Canada T1K 3M4
} 
symmetric group on the set $\{1, \ldots, k\}$. We denote by $\pi(x)$ or $\pi(X)$ the image of the element $x$ or subset $X$ under the permutation $\pi$. If $G$ is a permutation group on the set $\Omega$ and $X$ is a subset of $\Omega$ then $G_{X}$ denotes the set stabilizer of $X$ in $G$, i.e. $G_{X}=\{g \in G \mid g(X)=X\}$. If $\leq$ is a linear order in $\Omega$ and $X$ is a subset of $\Omega$ then we shall denote by $\min _{\leq} X$ the minimal element of $X$ in $(\Omega, \leq)$. Moreover, in the case that $\alpha \leq \beta$ and $\alpha \neq \beta$ we will write $\alpha<\beta$. If $\sigma$ is a permutation in $\Omega$ then it defines a linear order $\leq_{\sigma}$, where $\alpha \leq_{\sigma} \beta$ if and only if $\sigma^{-1}(\alpha) \leq \sigma^{-1}(\beta)$. The minimum element of $X$ with respect to $\leq_{\sigma}$ will be denoted by $\min _{\leq \sigma}(X)$.

Let $\mathscr{S}$ be a set of permutations of $\Omega$, Pr be a probability distribution on $\mathscr{S}$ and $k$ be a natural number. $\mathscr{S}$ is called a $k$-min-wise independent family, $k$-MWI for short, if

$$
\operatorname{Pr}(\min \pi(X)=\pi(x))=\frac{1}{|X|}
$$

for any $X \subseteq \Omega$ such that $|X| \leq k$ and for any $x \in X$. This definition was motivated by applications in computer science. In fact such a family is important in algorithms used in practice by software to find duplicate documents, see [3]. Later, such sets were applied in other contexts such as derandomization of algorithms. We say that $G$ is a $k$-MWI group if $G$ is a $k$-MWI family and $G$ is a permutation group of $\Omega$.

In this paper we consider exclusively $k$-MWI families $\mathscr{S}$ for the uniform distribution. In [1] Theorem 3.1, it has been proved that if $G$ is a $k$-MWI group with respect to some probability distribution $\operatorname{Pr}$ then $G$ is $k$-MWI with respect to the uniform distribution. Therefore dealing with $k$-MWI groups our assumption is not at all a restriction.

We begin with a definition.

Definition 1 We say that a set of permutations $\mathscr{S}$ is locally $k$-MWI, $k \geq 1$, if for every subset $X$ of size at most $k, \tau \in \mathscr{S}$ and for every $x \in X, y \in \tau(X)$ we have that

$$
\frac{|\{\pi \in \mathscr{S} \mid \pi(X)=\tau(X), \pi(x)=y\}|}{|\{\pi \in \mathscr{S} \mid \pi(X)=\tau(X)\}|}=\frac{1}{|X|} .
$$

Our main result is the following:

Theorem 1 Let $\mathscr{S}$ be a set of permutations of $\operatorname{Sym} \Omega$ and $k$ be a natural number. $\mathscr{S}$ is a k-MWI family with respect to any linear order and with respect the uniform distribution if and only if $\mathscr{S}$ is locally $k-M W I$. 
As a consequence of this theorem we prove a complete classification of the $k$-MWI groups with respect to any linear order in the underlying set $\Omega$, for $k \geq 3$.

In the next section we give the proof of Theorem 1. Then we outline the classification of groups with this property, and discuss some further directions.

\section{Proof of Theorem 1}

Let $\Omega$ and $\mathscr{S}$ be as in the statement of the theorem. First we prove the forward direction. So suppose that $\mathscr{S}$ is $k$-MWI with respect to any linear ordering of $\Omega$. Without loss of generality we may assume that $\Omega=\{1, \ldots, n\}$.

Choose $h \leq k$. Let $A=\{1, \ldots, h\}, B=\{2, \ldots, h\}$, and set $\mathscr{F}=\{X \subseteq \Omega|| X \mid=$ $h-1\}$. Now define a non-simple bipartite graph $\Gamma$ : the vertex set of $\Gamma$ is $\Omega \cup \mathscr{F}$; for each $\pi \in \mathscr{S}$, there is an edge joining $\pi(1) \in \Omega$ to $\pi(B) \in \mathscr{F}$.

For $\tau \in \mathscr{S}, i \in A$, and $Y \subseteq A$, let us denote by $f_{\tau}(i, Y)$ the number of edges $(i, X)$ of $\Gamma$ such that $X \cap \tau(A)=Y$, where $i \in \tau(A)$ and $Y \subseteq \tau(A)$.

Fix $\tau$ in $\mathscr{S}$, and pick $\sigma$ in $\operatorname{Sym}(h)$ (the subgroup of $\operatorname{Sym}(n)$ fixing $\{h+$ $1, \ldots, n\}$ pointwise) and $\tau \sigma(i)$ in $\tau(A)$. The number of permutations $\pi$ in $\mathscr{S}$ having $\pi(1)=\tau \sigma(i)$ as $\leq \tau \sigma$-minimum of the set $\pi(A)$ is the number of edges $(\tau \sigma(i), \tau \sigma(X))$ in $\Gamma$ such that $\tau \sigma(i)<_{\tau \sigma} \tau \sigma(X)$. By definition of $\leq_{\tau \sigma}$, this means $i<X$, and, as $i \in A$, this is equivalent to $i<X \cap A$. Summing, we have

$$
\left|\left\{\pi \in \mathscr{S} \mid \min _{\leq \tau \sigma} \pi(A)=\pi(1)=\tau \sigma(i)\right\}\right|=\sum_{Y \subseteq\{i+1, \ldots, h\}} f_{\tau}(\tau \sigma(i), \tau \sigma(Y)) .
$$

Now, $\mathscr{S}$ is a $k$-MWI family with respect to any linear order on $\Omega$. Therefore we have

$$
\begin{aligned}
\frac{|\mathscr{S}|}{h}= & \left|\left\{\pi \in \mathscr{S} \mid \min _{\leq \tau \sigma} \pi(A)=\pi(1)\right\}\right| \\
= & \sum_{i=1}^{h} \sum_{Y \subseteq\{i+1, \ldots, h\}} f_{\tau}(\tau \sigma(i), \tau \sigma(Y)) \\
& \quad+\left|\left\{\pi \in \mathscr{S} \mid \min _{\leq \tau \sigma} \pi(A)=\pi(1), \pi(1) \notin \tau(A)\right\}\right| .
\end{aligned}
$$

We claim that the second summand in (1) does not depend on $\sigma \in \operatorname{Sym}(h)$. Indeed, let $\pi$ be a permutation in $\mathscr{S}$ such that $\min _{\leq_{\tau \sigma}} \pi(A)=\pi(1)$ and $\pi(1) \notin \tau(A)$. We get $\min _{\leq \sigma} \tau^{-1} \pi(A)=\tau^{-1} \pi(1)$ and $\tau^{-1} \pi(1) \notin A$. Now, $\sigma$ is a permutation stabilizing the set $A$ and acting trivially on $\Omega \backslash A$; therefore we have $\min _{\leq} \tau^{-1} \pi(A)=$ $\tau^{-1} \pi(1)$. This proves our claim. In particular, from equation (1) we have that 


$$
Q(\sigma)=\sum_{i=1}^{h} \sum_{Y \subseteq\{i+1, \ldots, h\}} f_{\tau}(\tau \sigma(i), \tau \sigma(Y))
$$

is a constant that does not depend on the choice of $\sigma$ in $\operatorname{Sym}(h)$.

We claim that $f_{\tau}(\tau(i), \tau(Y))=f_{\tau}(\tau \sigma(i), \tau \sigma(Y))$ for every $\sigma \in \operatorname{Sym}(h)$ such that $\sigma(Y \cup\{i\})=Y \cup\{i\}$. We prove this by induction on $|Y|$. Assume $|Y|=1$. Let $1 \leq i<j \leq h$ and $\sigma$ be a permutation of $\operatorname{Sym}(h)$ mapping $i$ into $h-1$ and $j$ into $h$. Using the definition of $\Gamma$ we get

$$
0=Q(\sigma)-Q((h-1, h) \sigma)=f_{\tau}(\tau(i),\{\tau(j)\})-f_{\tau}(\tau(j),\{\tau(i)\}) .
$$

Therefore $f_{\tau}(\tau(i),\{\tau(j)\})=f_{\tau}(\tau(j),\{\tau(i)\})$. Assume the result for $|Y|=l-1$ and let us prove it for $|Y|=l$. Let $1 \leq i_{l+1}<\cdots<i_{2}<i_{1} \leq h$ and $\sigma$ be a permutation mapping $i_{j}$ into $h-j+1$. Consider the permutation $\eta=(h-l, \ldots, h-1, h)$. Now, using the inductive hypothesis we have

$$
\begin{aligned}
0 & =Q(\sigma)-Q(\eta \sigma) \\
& =\sum_{i=h-l Y \subseteq\{h-l+1, \ldots, h\}}^{h}\left(f_{\tau}(\tau \sigma(i), \tau \sigma(Y))-f_{\tau}(\tau \eta \sigma(i), \tau \eta \sigma(Y))\right) \\
& =f_{\tau}\left(\tau\left(i_{l+1}\right), \tau\left(\left\{i_{l}, \ldots, l_{1}\right\}\right)\right)-f_{\tau}\left(\tau\left(i_{l}\right), \tau\left\{i_{l-1}, \ldots, i_{1}, i_{l+1}\right\}\right) .
\end{aligned}
$$

Similarly, using $\eta^{l-j+1}$ rather than $\eta$, we have

$$
f_{\tau}\left(\tau\left(i_{j}\right), \tau\left(Y-\left\{i_{j}\right\}\right)\right)=f_{\tau}\left(\tau\left(i_{l+1}\right), \tau\left(Y-\left\{i_{l+1}\right\}\right)\right)
$$

for every $j$, where $Y=\left\{i_{l+1}, \ldots, i_{1}\right\}$.

Now we are ready to prove the forward implication in the theorem. By the previous discussion, $f(\tau(1), \tau(B))=f(\tau \sigma(1), \tau \sigma(B))$ for every $\sigma \in \operatorname{Sym}(h)$. This proves that, for every $x$ in $\tau(A)$, the number of elements in $\mathscr{S}$ such that $\pi(1)=x$ and $\pi(A)=\tau(A)$ equals the number of elements such that $\pi(1)=\tau(1)$ and $\pi(A)=\tau(A)$. Therefore we are done.

For the reverse implication, assume that $\mathscr{S}$ is locally $k$-MWI. Let $h \leq k$ and let $X$ be an $h$-set of $\Omega$ and $x \in X$. Let us denote by $\Sigma$ the set $\{\pi(X) \mid \pi \in \bar{S}\}$. We have

$$
\begin{aligned}
|\{\pi \in \mathscr{S} \mid \min \pi(X)=\pi(x)\}| & =\sum_{Y \in \Sigma} \mid\{\pi \in \mathscr{S}|\pi(X)=Y, \min Y=\pi(x)|\} \\
& =\sum_{Y \in \Sigma} \frac{|\{\pi \in \mathscr{S} \mid \pi(X)=Y\}|}{|X|}=\frac{|\mathscr{S}|}{|X|},
\end{aligned}
$$


so the theorem has been proved. We note that this direction of the proof was given in [5], Lemma 2, in the case where $\mathscr{S}$ is a group.

\section{A consequence of Theorem 1}

Corollary 1 Let $G$ be a finite permutation group on the set $\Omega$. Then $G$ is a $k-M W I$ group with respect to any linear order in $\Omega$ if and only if for every subset $X$ of $\Omega$ of size at most $k$ we have that $G_{X}$ is transitive on $X$.

Proof This is immediate from Theorem 1.

We note that if, for every subset $X$ of $\Omega$ of size $k$, the group $G_{X}$ is transitive on $X$, then $G$ is $(k-1)$-homogeneous. In fact, let $A$ and $B$ be $(k-1)$-sets. Assume that $A \cap B$ is a $(k-2)$-set. Then $A$ and $B$ lie in the same $G$-orbit. For if $X=A \cup B$ then $A=X \backslash\{b\}$ and $B=X \backslash\{a\}$, for some $a \in A$ and $b \in B$. Now, $X$ is a $k$-set, so by hypothesis, $G_{X}$ contains an element mapping $a$ into $b$, and so, $A$ into $B$. With an easy induction on $|A \cap B|$ and with a connectedness argument we get that all $(k-1)$-sets are in the same orbit.

This remark allow us to get the following classification.

Theorem 2 Let $G$ be a finite permutation group on the set $\Omega$ and let $k$ be a positive integer with $k \geq 3$. Then the following conditions are equivalent:

(a) $G$ is a k-MWI group with respect to any linear order on $\Omega$;

(b) $G_{X}$ is transitive on $X$ for any subset $X$ of $\Omega$ with $|X| \leq k$;

(c) $G$ is one of the groups from Table 1.

Proof (Sketch) Corollary 1 shows that (a) and (b) are equivalent. We have to show that (b) and (c) are equivalent.

Assume that (b) holds. Then $G$ is $h$-homogeneous for any $h<k$ (in particular $G$ is 2-homogeneous). Now, apart known exceptions, if $G$ is a $h$-homogeneous group with degree $n$, for $h \leq n / 2$, then $G$ is $h$-transitive. The list of all possible exceptions can be found in [4]. Thus the proof of Theorem 2 is a case-by-case analysis among the list of 2-transitive groups and the list of groups in [4].

In this analysis, the following remark is useful. 
Suppose that $G$ is a $t$-transitive permutation group on $G$ and that all

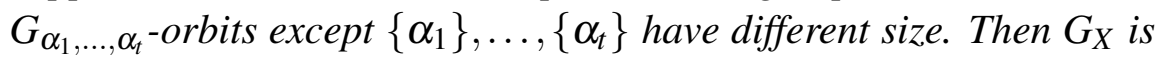
transitive on $X$ for any subset $X$ of $\Omega$ with $|X| \leq t+1$. In particular $G$ is $(t+1)-M W I$.

Using this tool, we can deal with the almost simple groups. For instance, $M_{22}$ is 3-transitive and the stabilizer of four distinct points has orbits of size $1,1,1,3,16$. Therefore, $M_{22}$ is 4-MWI with respect to any linear order. Furthermore, $M_{22}$ is not 4-homogeneous, therefore $M_{22}$ can not be 5-MWI with respect to all linear orders.

The analysis of the affine 2-transitive groups requires other remarks. We present and prove the main ingredient of this classification.

Let $G$ be an affine 2-transitive group on $V, V$ an n-dimensional $\mathbb{F}_{q^{-}}$ vector space, $q=p^{m}$. If $G$ is a 3-MWI group with respect to any linear order then $q=2,3,4$ or $q=8$. In particular, if $q=8$ then $G$ contains the Galois group of $\mathbb{F}_{8}$.

To prove this, assume that $q>2$. By Corollary $1, G_{X}$ is transitive on $X$ for any $X \subseteq V$ of size 3. Fix $\left(e_{i}\right)_{i}$ a basis of $V, a \in \mathbb{F}_{q} \backslash\{0,1\}$ and $X=\left\{0, e_{1}, a e_{1}\right\}$. The group $G_{X}$ is transitive on $X$ if and only if it contains an element $\varphi: \xi \mapsto A \xi^{\sigma}+v$ such that $\varphi(0)=e_{1}, \varphi\left(e_{1}\right)=a e_{1}$ and $\varphi\left(a e_{1}\right)=0$. This proves that for all $a \in$ $\mathbb{F}_{q} \backslash\{0,1\}$ there exists $\sigma \in \operatorname{Aut}\left(\mathbb{F}_{q}\right)$ such that $a^{\sigma+1}-a^{\sigma}+1=0$. In particular any $a \in \mathbb{F}_{q} \backslash\{0,1\}$ is a root of $X^{p^{i}+1}-X^{p^{i}}+1$ for some $i$. This yields that the characteristic of $\mathbb{F}_{q}$ is either 2 or 3 .

Assume that $q=3^{m}$. The equation $X^{3^{i}+1}-X^{3^{i}}+1$ has at most $3^{i}+1$ roots. Therefore summing on all the equations we have $\sum_{i=0}^{m-1}\left(3^{i}+1\right) \geq 3^{m}-2$. This happens if and only if $m=1$.

Consider the case $q=2^{m}$. Now, let us study the solutions of the equation $X^{2^{m-1}+1}+X^{2^{m-1}}+1$ in $\mathbb{F}_{q}$. We have $0=X^{2^{m}}+X=X^{-2}\left(X^{2^{m-1}+1}\right)^{2}+X=X^{-2}\left(X^{2^{m}}+\right.$ $1)+X=X+X^{-1}+X^{-2}$, if and only if $X^{3}+X+1=0$. Therefore $X^{2^{m-1}+1}+X+1$ has at most 3 solutions in $\mathbb{F}_{q}$. This yields $\sum_{i=0}^{m-2}\left(2^{i}+1\right)+3 \geq 2^{m}-2$. This happens if and only if $q=2,4$ or $q=8$. Now the remaining part is easy to achieve.

Further details of the classification may be obtained from the second author. In Table $1, C$ denotes the Galois group of $\mathbb{F}_{8}$ over $\mathbb{F}_{2}$.

For $k=2$, no complete classification exists. The groups which are 2-MWI for every linear order are just those transitive groups for which every pair of points 
is interchanged by some group element. These groups are sometimes referred to as generously transitive, and have the property that the permutation character is multiplicity-free (so they are examples of Gelfand pairs), in which all irreducible constituents are real. See Saxl [6], for example.

\section{Concluding remarks}

For practical purposes it is often necessary to get a small $k$-MWI family. In other words, for fixed $\Omega$ and $k$, the complexity of the algorithms using MWI families is strictly related to the size of the family. So clearly the problem consists in finding a compromise between $k$ and the size of the family $\mathscr{S}$. From Theorem 1 we realize that if the family has to be $k$-MWI with respect to any linear order then the actual size has to be comparatively big. In particular, it is worth noting that if $G$ is a $k$-MWI group with respect to any linear order and $k \geq 7$ then $G$ has to contain the alternating group $\operatorname{Alt}(\Omega)$, see Theorem 2. Therefore it is reasonable to look at particular orders of the underlying set. Bargachev [2] has shown that there are 4-MWI groups of degree $n$ and size $O\left(n^{2}\right)$. From Table 1 we see that the order of a 4-MWI group with respect to any linear order and degree $n$ has to be at least $\Omega\left(n^{3}\right)$.

Next we present a variant of this problem. We say that the family $\mathscr{S}$ is $(\varepsilon, k)$ MWI if

$$
\frac{1}{|X|(1+\varepsilon)} \leq \operatorname{Pr}(\min \pi(X)=\pi(x)) \leq \frac{1}{|X|(1-\varepsilon)}
$$

for every subset $X$ of $\Omega$ of size at most $k$ and for every $x \in X$. Here $k$ is a positive integer and $\varepsilon \geq 0$. One might hope that for "small" values of $\varepsilon$ the variety of families that arise is considerably richer than the previous ones. Also, we remark that a group $G$ is $(\varepsilon, k)$-MWI with respect to some probability distribution $\operatorname{Pr}$ then $G$ is $(\varepsilon, k)$-MWI with respect to the uniform distribution. The proof of this result is exactly the same as Theorem 3.1 in [1].

Also, mimicking Definition 1 one can define a local approximated version: indeed, a set of permutations $\mathscr{S}$ is locally $(\varepsilon, k)$-MWI, $k \geq 1$, if for every subset $X$ of size at most $k, \tau \in \mathscr{S}$ and for every $x \in X, y \in \tau(X)$ we have that

$$
\frac{1}{|X|(1+\varepsilon)} \leq \frac{|\{\pi \in \mathscr{S} \mid \pi(X)=\tau(X), \pi(x)=y\}|}{|\{\pi \in \mathscr{S} \mid \pi(X)=\tau(X)\}|} \leq \frac{1}{|X|(1-\varepsilon)}
$$

Clearly, if $\mathscr{S}$ is a locally $(\varepsilon, k)$-MWI family then $\mathscr{S}$ is $(\varepsilon, k)$-MWI with respect to any linear order, see the last paragraph of the proof of Theorem 1. A 
permutation group $G$ which is locally $(\varepsilon, k)$-MWI for any $\varepsilon<1$ is $k$-MWI, by the equivalence of (a) and (b) in Theorem 2.

Finally, we remark that every elementary abelian 2-group $G$, acting regularly, is $\left(\frac{1}{3}, 3\right)$-MWI with respect to any order. For take a 3 -set $X=\{\alpha, \beta, \gamma\}$, and let $\delta \in \Omega$ be the point such that the stabilizer $G_{Y}$ of $Y=\{\alpha, \beta, \gamma, \delta\}$ has order 4. It is easy to prove that for every $\sigma \in G$ we have

$$
\left|\left\{\pi \in G_{Y} \mid \min \sigma \pi(X)=\sigma \pi(\alpha)\right\}\right| \in\{1,2\} .
$$

Summing over a transversal of $G_{Y}$ in $G$ we have that $G$ is $\left(\frac{1}{3}, 3\right)$-MWI with respect to any linear order. The size of $G$ is $n=|\Omega|$; a group which is 3-MWI with respect to any order has size at least $n(n-1) / 2$. On the other hand, this group is not locally $(\varepsilon, 3)$-MWI for any $\varepsilon<1$, since the stabiliser of a 3 -set acts trivially on it.

\section{References}

[1] V. Bargachev, Some properties of min-wise independent families and Groups of permutations, Zap. Nauchn. Sen. S.-Peterburg. Otdel. Mat. Inst. Steklov 316 (2004), 30-41, 224-225.

[2] V. Bargachev, personal communication.

[3] A. Z. Broder, M. Charikar, A. M. Frieze and M. Mitzenmacher, Min-wise independent permutations, J. Comput. Syst. Sci. 60, (2000), 630-659.

[4] J.D.Dixon and B.Mortimer, Permutation Groups, Springer, (1996).

[5] C. Franchi and M. Vsemirnov, Min-wise independent groups, European Journal of Combinatorics 24, (2003), 630-659.

[6] J. Saxl, On multiplicity-free permutation representations, in Finite Geometries and Designs (ed. P. J. Cameron, J, W. P. Hirschfeld and D. R. Hughes), pp. 337-353, London Math. Soc. Lecture Notes 49, Cambridge Univ. Press, Cambridge, 1981. 
Table 1: The $k$-MWI groups with respect to any order $(k \geq 3)$

\begin{tabular}{|c|c|c|}
\hline$G$ & Condition & $(|\Omega|, k)$ \\
\hline $\operatorname{Alt} \Omega \leq G \leq \operatorname{Sym} \Omega$ & $|\Omega| \geq 4$ & $(|\Omega|,|\Omega|)$ \\
\hline$M_{12}$ & & $(12,6)$ \\
\hline$M_{24}$ & & $(24,6)$ \\
\hline$M_{11}$ & & $(11,5)$ or $(12,4)$ \\
\hline$M_{23}$ & & $(23,5)$ \\
\hline$M_{22} \leq G \leq \operatorname{Aut} M_{22}$ & & $(22,4)$ \\
\hline $\operatorname{PSL}(n, q) \leq G \leq \operatorname{P\Gamma L}(n, q)$ & $n \geq 3$ & $\left(\left(q^{n}-1\right) /(q-1), 3\right)$ \\
\hline $\operatorname{PGL}(2, q) \leq G \leq \operatorname{P\Gamma L}(2, q)$ & $q \neq 4,5,7$ & $(q+1,4)$ \\
\hline $\operatorname{PSL}(2, q) \leq G \leq \operatorname{P} \Sigma \mathrm{L}(2, q)$ & $q \neq 4,7$ & $(q+1,3)$ \\
\hline $\operatorname{PSL}(2,7) \leq G \leq \operatorname{PGL}(2,7)$ & & $(8,4)$ \\
\hline $\operatorname{PGL}(2,5)$ & & $(6,6)$ \\
\hline $\operatorname{PSL}(2,11)$ & & $(11,3)$ \\
\hline $\operatorname{Alt}(7)$ & & $(15,3)$ \\
\hline$H S$ & & $(176,3)$ \\
\hline $\mathrm{Co}_{3}$ & & $(276,3)$ \\
\hline $\operatorname{Sp}(2 d, 2)$ & $d \geq 3$ & $\left(2^{2 d-1}+2^{d-1}, 3\right)$ \\
\hline $\operatorname{Sp}(2 d, 2)$ & $d \geq 3$ & $\left(2^{2 d-1}-2^{d-1}, 3\right)$ \\
\hline $\operatorname{PGU}(3, q) \leq G \leq \operatorname{P\Gamma U}(3, q)$ & & $\left(q^{3}+1,3\right)$ \\
\hline $\mathrm{A} \Gamma \mathrm{L}(1, q)$ & $q=3,8$ & $(q, 3)$ \\
\hline $\operatorname{ASL}(n, q) \leq G \leq \operatorname{A\Gamma L}(n, q)$ & $q=3,4 ; n \geq 2$ & $\left(q^{n}, 3\right)$ \\
\hline $\operatorname{ASL}(n, 2)$ & $n \geq 2$ & $\left(2^{n}, 4\right)$ \\
\hline $\mathrm{A} \Sigma \mathrm{L}(n, 8) \leq G \leq \mathrm{A} \Gamma \mathrm{L}(n, 8)$ & $n \geq 2$ & $\left(8^{n}, 3\right)$ \\
\hline$V \rtimes \operatorname{Alt}(6)$ & & $(16,3)$ \\
\hline$V \rtimes \operatorname{Alt}(7)$ & & $(16,4)$ \\
\hline$V \rtimes \operatorname{PSU}(3,3)$ & & $(64,3)$ \\
\hline$V \rtimes G_{2}(q) \unlhd G$ & $q=2,4$ & $\left(q^{6}, 3\right)$ \\
\hline$V \rtimes\left(G_{2}(8) \cdot C\right) \unlhd G$ & & $\left(8^{6}, 3\right)$ \\
\hline$V \rtimes \mathrm{Sp}(2 d, q) \unlhd G$ & $q=2,3,4 ; d \geq 3$ & $\left(q^{2 d}, 3\right)$ \\
\hline$V \rtimes(\operatorname{Sp}(2 d, 8) \cdot C) \unlhd G$ & $d \geq 3$ & $\left(8^{2 d}, 3\right)$ \\
\hline
\end{tabular}

